\title{
Consumer demand for time of use electricity tariffs: A systematized review of the empirical evidence
}

\author{
Moira L. Nicolson*, Michael J. Fell, Gesche M. Huebner \\ UCL Energy Institute, Central House, 14 Upper Woburn Place, London WC1H ONN, UK
}

\section{A R T I C L E I N F O}

\section{Keywords:}

Demand-side response

Meta-analysis

Time of use tariff

Consumer acceptance

\begin{abstract}
A B S T R A C T
Time of use (TOU) tariffs, if widely adopted, could help make electricity more secure, clean and affordable. However, quite little is known about whether consumers will switch to a TOU tariff or what might increase uptake if switching rates are lower than required. This paper presents the results of a systematized review and meta-analysis combining the results of 66 measures of uptake to a variety of TOU tariffs across 27 studies conducted in six countries. It provides the first robust estimate of consumer demand and correlates of demand for TOU tariffs that is not based on the results from just a single study or tariff. Four main conclusions emerge. First, if consumers are left to opt-in to TOU tariffs, uptake could be as low as $1 \%$ unless efforts are made to close the intention-action gap, otherwise enrolment could reach $43 \%$. Second, if enrolment is opt-out, uptake could approach $100 \%$. Third, whilst national surveys indicate the potential appetite for TOU tariffs in a population, they are insufficient for predicting future TOU tariff adoption rates; the median proportion of domestic energy bill payers who say they would be willing to switch to a TOU tariff in national surveys is five times higher than the median enrolment rate to TOU tariffs offered by utilities. Fourth, real-time pricing tariffs, in which the price of electricity varies freely throughout the day, are less popular than static TOU tariffs which have fixed peak and off-peak rates. This paper discusses the limitations of opt-out enrolment for TOU tariffs and presents results suggesting that small upfront payments, bill protection and automation are promising alternative methods of increasing opt-in enrolment. Policymakers and researchers should now consider how recruitment will be performed, weighing up the benefits to society as a whole against the distributional impacts for individuals and groups.
\end{abstract}

\section{Introduction}

Encouraging domestic consumers to change the time of day at which they use electricity is a key part of many governments' plans to ensure national energy supplies are secure and affordable in the transition towards greater penetration of intermittent renewable energy sources and the electrification of heat and transport [1]. One way in which consumers will be incentivised to change their consumption patterns is through price signals delivered via time of use (TOU) electricity tariffs, in which the price of electricity varies depending on factors such as electricity network constraints and the wholesale price of electricity. A large body of literature demonstrates that consumers will alter their consumption patterns in response to a range of TOU tariffs, with an average reduction in peak time energy consumption of around 15\% depending on the tariff design [2], see also [3,4] and, in particular, [5] which reviews 30 trials on the impact of TOU tariffs on electricity demand. An underlying assumption of many of these studies, including government decarbonisation strategies, is that consumers will voluntarily sign up to a TOU tariff in the first place. However, the evidence on level of consumer demand for TOU tariffs is far less clear.

Whilst TOU electricity pricing has been an established part of grid management strategies involving large industrial and commercial users for many years, domestic TOU programmes remain restricted to relatively basic legacy options such as Economy 7 tariffs [6] in Great Britain (GB), or the Tempo Tariff in France [7]. Aside from the United States, where more modern TOU tariffs are now commercially available, there

\footnotetext{
Abbreviations: CPP, Critical peak pricing; CPR, Critical peak rebate; DP, Dynamic pricing; EU, European Union; GB, Great Britain; OECD, Organisation for Economic

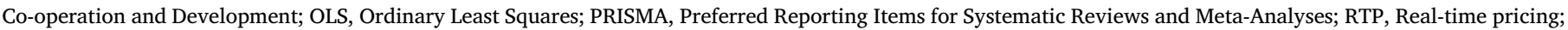
TOU, Time of use; UK, United Kingdom; US, United States

* Corresponding author.

E-mail address: m.nicolson.11@ucl.ac.uk (M.L. Nicolson).
} 
is therefore no useful measure of current commercial consumer demand for TOU tariffs. ${ }^{1}$ Alternative sources of evidence on consumer demand for TOUs include recruitment rates into TOU field trials and measures of stated demand elicited from survey participants. However, to our knowledge, there has been no attempt to synthesise the evidence from this wide range of sources to provide an overall estimate of the likely uptake of TOUs amongst domestic energy bill payers.

Further, if consumer uptake is lower than required - as our initial analysis of consumer behavior in the energy market suggests it could be - evidence is also required on what recruitment strategies and enabling technologies are likely to be most effective at increasing uptake. For example, in the behavioural science literature, a large body of research documents the way in which enrolment rates to various services and products are higher when people are enrolled automatically, with the option to unenroll ('opt-out'), as opposed to when people must take active steps to sign up ('opt-in'). Notable examples are workplace pension schemes, green energy tariffs and organ donor registration [8-11]. Some TOU tariff field trials enrolled participants onto TOU tariffs by default, unless consumers opted-out, whereas others relied on consumers actively deciding to opt-in, presenting an opportunity to understand which method may be more successful at stimulating uptake. In addition, some studies provided participants with assistive technologies, usually smart thermostats that customers could programme to lower set-points during the higher peak time price periods. Such technologies may also make TOU tariffs more, or even less, desirable, depending on how these technologies are perceived. Moreover, there are many different types of tariffs for which the price of electricity varies throughout the day, and some tariff designs may be more appealing to consumers than others. Five key time-varying tariff designs are:

- Static TOU. Prices vary during the day in a fixed and regular way, for example by having a peak price between 4 and 8 p.m. on weekdays, and an off-peak price at other times.

- Dynamic TOU (DP). Price points are fixed, but the times at which they apply vary from day to day. For example, there may be low, medium, and high price periods, and customers are notified in advance between which times those prices will apply,

- Real-time pricing (RTP). Prices vary in real-time (e.g. to the hour or half hour) depending on the current wholesale cost of electricity.

- Critical peak pricing (CPP). Pricing is mostly flat, but there are occasional high price 'events' of which customers are notified in advance.

- Critical peak rebates (CPR), also known as peak time rebates. Pricing is flat, but at certain times (notifiable in advance) customers are rewarded for reducing their electricity demand compared to some agreed amount.

For simplicity, this paper adopts the convention of using the term 'time of use' tariff (here, abbreviated to TOU tariff) as a generic term to refer to a whole spectrum of time-varying tariffs, including both static and dynamic pricing options. ${ }^{2}$

This paper presents the design and results of a systematized literature review [12] aimed at answering five main review questions:

\section{- How much domestic consumer demand is there for TOU tariffs?}

\footnotetext{
${ }^{1}$ We exclude countries such as Italy which have made time of use tariffs mandatory since mandatory enrolment rates do not provide evidence of consumer demand.

${ }^{2}$ Although this is a conventional use of the terminology, sometimes the term 'time of use' tariff (here, abbreviated to TOU tariff) is used to refer to a specific type of time-varying tariff design that has a peak and off-peak price at the same time of the day or week; following the conventions in the literature on demandside response, we refer to this sub-group of TOU tariffs as a static TOU tariff (see bulleted list).
}

- Does domestic consumer demand for TOU tariffs vary by tariff design?

- Does domestic consumer demand for TOU tariffs vary according to the presence of automation technologies?

- Does domestic consumer demand for TOU tariffs vary by the way in which the tariff is framed to consumers, such as whether the choice is opt-in rather than opt-out?

- Is there cross-country variation in demand for TOU tariffs?

This systematized review uses methods from a systematic review, including the use of a review protocol in which the search strategy, inclusion/exclusion criteria and extraction methods are determined in advance. However, like a rapid review, the completeness of searching was determined by resource constraints [12]. This review also includes a meta-analysis, "which statistically combines the results of quantitative studies to provide a more precise estimate of the results" [12], p. 94 to answer the research questions above. The review provides evidence from six countries, covering Australia, France, Norway, Netherlands, United Kingdom and the United States.

The rest of this paper is structured as follows. Section 2 outlines the method used to conduct the systematized review, including the search strategy and screening criteria as well as the extraction and synthesis of the uptake measures. Section 3 presents the results of the search and synthesis used to obtain a measure of overall uptake and uptake by country, tariff design, framing etc. in a meta-analysis. Section 4 discusses the strength of the evidence for consumer demand for TOU tariffs in light of the limitations of the original study designs and the reviewlevel limitations (e.g. incomplete retrieval of relevant research) before concluding, in Section 5, with an overall recommended 'best' estimate of consumer demand for TOU tariffs and the key research gaps. The reporting of the design and results follows the PRISMA check-list [13] for reporting items for systematic reviews (mostly used in medical research) as closely as possible.

\section{Method - a systematized review}

\subsection{Criteria for considering studies for this review}

A review protocol was prepared in advance of conducting the review (see additional online material). We included studies written in the English language that document empirical, quantitative findings on switching rates to commercially available tariffs or hypothetical sign up rates, elicited in surveys, to TOU tariffs amongst domestic energy consumers in Organisation for Economic Co-operation and Development (OECD) countries.

The following types of report were excluded:

- Studies that documented qualitative findings only, because these types of studies could not be used to provide a quantitative measure of uptake.

- Studies that did not report empirical results (e.g. include only modelled uptake), because these studies would either be reliant on an empirical measure which our inclusion criteria would capture or would be based on targets or estimated optimum uptake levels, neither of which are equivalent to actual consumer demand.

- Studies that did not report research including a TOU (e.g. which focused only on direct load control or other non price-based demand-side response).

- Studies focused exclusively on the non-domestic sector.

- Studies reporting work conducted in non-OECD countries, because it was judged that such countries may have different priorities and concerns related to electricity usage (e.g. in developing countries, particularly energy access) that would make such research better suited to a separate review.

Studies reporting uptake measures based only on study recruitment 
Table 1

Search terms used in conducting the search with example search string for use in Scopus.

\begin{tabular}{|c|c|c|}
\hline & Time of use & Uptake \\
\hline \multirow[t]{8}{*}{ Concept } & Time of use tariffs & Uptake \\
\hline & Time-varying tariffs & Consumer \\
\hline & Off peak tariffs & Acceptability/acceptance \\
\hline & Dynamic pricing & Switching \\
\hline & Cost-reflective tariffs & Preferences \\
\hline & Critical peak pricing/rebates & \\
\hline & Peak-time rebates & \\
\hline & Real-time pricing & \\
\hline \multirow[t]{9}{*}{ Search term } & "time of use" & uptake \\
\hline & "time-of-use" & consumer* \\
\hline & "time-varying" & accept* \\
\hline & "off peak" & switch* \\
\hline & $\begin{array}{l}\text { dynamic W/2 pric* OR } \\
\text { tariff* }\end{array}$ & preference* \\
\hline & "cost-reflective" & \\
\hline & "critical peak" & \\
\hline & "peak-time"/peaktime & \\
\hline & "real-time pric*"/realtime & \\
\hline Scopus & \multirow{2}{*}{\multicolumn{2}{|c|}{$\begin{array}{l}\text { TITLE-ABS-KEY("time of use" OR "time-of-use" OR "time-varying" } \\
\text { OR "off peak" OR (dynamic W/2 pric* OR tariff*) OR "cost- } \\
\text { reflective" OR "critical peak" OR "peak-time" OR peaktime OR "real- } \\
\text { time pric*" OR "realtime pric*") AND TITLE-ABS-KEY(uptake OR } \\
\text { consumer* OR accept* OR switch* OR preference*) AND ALL(tariff } \\
\text { OR pric*) AND ALL (energy OR electr*) }\end{array}$}} \\
\hline example & & \\
\hline
\end{tabular}

\section{- IEEE Xplore}

Searches were also developed based on the above search terms for the websites of the following organisations:

- UK Department for Business, Energy \& Industrial Strategy

- Ofgem (GB energy regulator)

- Citizens Advice (GB consumer body)

- Sustainability First

- National Grid

- European Commission Research and Innovation (Energy)

- US Department of Energy (including SciTech Connect)

- Websites of UK and US academic institutions (URLs including ".ac.uk" and ".edu")

- ECEEE and ACEEE summer study proceedings

The focus on US organisations was motivated on the basis that the US has a relatively advanced market in demand-side response and, based on past research, were known to have undertaken a number of relevant studies that were not all published in academic journals. The additional focus on UK institutions was driven by a similar knowledge about the UK's research on consumer uptake to TOU tariffs as well as by the requirements of a wider project of which this review formed part. By choosing to focus the search on global bibliographic databases, UK, EU and US institutions and international conferences we aimed to maximize coverage given the availability of resources. These choices

Table 2

Inclusion and exclusion criteria for review screening.

\begin{tabular}{|c|c|}
\hline Include if source & Exclude if source \\
\hline Is in English & Is not in English \\
\hline Reports findings from empirical research or evaluation. & $\begin{array}{l}\text { Does not report empirical results (e.g. includes only modelled uptake), or } \\
\text { findings on uptake are based on study recruitment. }\end{array}$ \\
\hline Includes quantitative findings that can help to inform estimation of tariff uptake rates. & Reports only qualitative findings. \\
\hline $\begin{array}{l}\text { Reports research designed to enable estimation of the degree of consumers' expressed or } \\
\text { demonstrated willingness to sign up (hypothetically or in reality) to at least one TOU tariff } \\
\text { design, and the reasons associated with this. }\end{array}$ & $\begin{array}{l}\text { Does not report research including a TOU tariff (for example, focused only on } \\
\text { direct load control or other non-price-based demand response product). }\end{array}$ \\
\hline $\begin{array}{l}\text { Reports work conducted in an OECD country. } \\
\text { Is focused on the domestic sector. }\end{array}$ & $\begin{array}{l}\text { Reports work conducted in a non-OECD country. } \\
\text { Is focused on the non-domestic sector. }\end{array}$ \\
\hline
\end{tabular}

were also initially excluded (in the review protocol). However, the decision was later taken to include such studies where they aimed to recruit at a large scale (similar to a commercial product offering) in order to provide a greater range of evidence based on studies in which people are actually able to switch to the tariff having made the decision to switch (unlike in survey research). The full inclusion/exclusion criteria are summarised in Table A.1 in the appendix.

\subsection{Search methods for identification of studies}

An initial list of five recent publications on consumer demand for TOUs was identified [14-18] as a basis to generate keywords for electronic searches (Table 1). The reference lists of these publications were also checked and publications with titles that suggested they may fit the screening criteria were saved for further review. Forward citation checks were also conducted using Google Scholar to identify documents referencing these publications, which were saved for later review if the titles were deemed to fit the screening criteria above. Using a 'snowballing' approach, reference lists of documents that pass screening criteria were also accessed for inclusion.

The following bibliographic databases were searched:

- Scopus

- Web of Science (all databases)

- ACM Digital Library are considered when weighing up the external validity of the results.

Searches were recorded and reported to aid replicability (see additional online material) with potential sources saved in the reference management software Mendeley.

\subsection{Study selection}

Results were screened on the basis of title and abstract according to the inclusion and exclusion criteria outlined above (also summarised in Table 2). No exclusions were made on the basis of the design of the empirical study, for example, whether experimental or non-experimental. ${ }^{3}$ Screening was initially conducted in parallel by two screeners until high levels of agreement were reached in the EPPI-Reviewer software abstracts (review author initials: MF and GH]). Subsequent screening on title/abstract was performed by a single screener abstracts (review author initials: MF). Included items were then screened again on the full document. The list of final documents for inclusion were reviewed by the authors following screening, with publications known to be relevant but which were not present subjected to the same screening as above, and included if they passed the screening criteria.

\footnotetext{
${ }^{3}$ Whilst it would be standard practice to exclude studies without a control group in systematized reviews in the medical literature, since this paper is not assessing the causal effectiveness of an intervention, it was considered unnecessary to exclude studies on this basis.
} 


\subsection{Data extraction}

All sources included were coded in EPPI-Reviewer for the following key characteristics:

- Geographical location of study

- Whether air conditioning was a significant load

- Study start year

- Method of assessing uptake (survey, commercial product, trial recruitment, other)

- Experimental design

- Type(s) of TOU tariff(s) tested and their characteristics

- Organisation(s) administering the study

- Organisation(s) offering (or framed as offering) the TOU tariff(s)

- Characteristics of sample receiving the TOU intervention

- Size

- Sampling method - participant characteristics, recruitment method (opt-in, opt-out/framing)

- Whether an incentive was given to participants

- Whether bill protection was included

- Type(s) of outcome(s) measured by the intervention (including measure/proxy of uptake/responsiveness and customer satisfaction)

- Role of automating technology

- Whether an ongoing satisfaction assessment was conducted

- Reported outcome(s), key interpretations and main conclusions

Extraction was conducted by a single reviewer in EPPI-Reviewer abstracts (review author initials: MN). Not all studies reported uptake and so this had to be computed, where possible, from the information provided. ${ }^{4}$ Studies from which it was not possible to compute or obtain a measure of uptake were excluded at this point too. Report tables were compiled using EPPI-Reviewer in MS Word format from which a second extraction was undertaken to transpose key characteristics required for numerical analysis into MS Excel. ${ }^{5}$

\subsection{Data synthesis}

To compute an overall measure of uptake to TOU tariffs, uptake measures that were not already expressed as a proportion were converted into proportions in MS Excel. This involved converting measures obtained from Likert scale type responses into binary variables, in which the proportion of participants choosing a Likert scale point above neutral ${ }^{6}$ were coded as switchers.

Once the primary outcome variable was coded consistently across studies, a meta-analysis was undertaken. Meta-analysis is most commonly used to aggregate results of clinical trials and the standard definition of meta-analysis reflects this: "meta-analysis is a statistical methodology that integrates the results of several independent clinical trials that are considered by the analyst to be "combinable"" (Huque [1988] cited in Kontapantelis and Reeves [19]). Meta-analysis is a twostage process, the first of which involves providing an appropriate summary statistic for each study and the second in which the statistics are combined to obtain an overall average effect $[19,20]$.

\footnotetext{
${ }^{4}$ For example, a tariff trial might report the total number of participants solicited for participation under a description of recruitment strategy and also the total number of enrolled participants, when discussing the main effects of the tariff on energy consumption. Alternatively, a study might report the total number of customers enrolled on a commercially available tariff on offer to all French consumers, in which case the recruitment rate can be inferred from the population of France.

${ }^{5}$ Full extracted tables are available on request from the corresponding author.

${ }^{6}$ For example, on a 5-point Likert scale in which 1 is not willing to switch and 5 is strongly willing to switch, participants who selected 4 or 5 were coded as switchers.
}

For the first stage, we computed the mean and median uptake level for the overall sample and the mean uptake, with the lower and upper $95 \%$ confidence interval for the mean uptake, grouped by the following factors from our research questions: type of study, tariff design, country, framing, presence of bill protection, presence of automation and additional financial incentive (the latter factor excluded survey based measures, where incentives are used to compensate participants for the time taken to complete the survey and are not used to attract people onto the tariff itself). These summary statistics were obtained by importing the MS Excel file into the statistical software package Stata.

For the second stage, Ordinary Least Squares (OLS) ${ }^{7}$ regression was used to test whether there were statistically significant differences in uptake rates across these factors using the following equation:

$\gamma_{i s}=\alpha_{i s}+\beta_{i s} \theta_{s}+\beta_{i s} \mu_{i s}+\beta_{i s} \chi_{i s}+\delta_{s}+\varepsilon_{i s}$

where $\gamma_{i s}$ is a proportion ranging from 0.0 to 1.00 for each uptake measure $i$ in study $s$. The constant, $\alpha_{i s}$, equals the average uptake to a TOU tariff across each measure $i$ and study $s$ conditional on the covariates $\theta_{s}, \mu_{i s}$ and $\chi_{i s}$. The covariate $\theta_{s}$ is a dummy variable in which the value 1 is assigned to an uptake measure $i$ from a study $s$ that reports willingness to switch to a TOU tariff from a survey experiment and the value zero if the uptake measure is from a study that reports the participant recruitment rate into a TOU tariff trial or uptake to a commercially available tariff. This is included in all specifications because it is assumed that the method of measuring uptake will affect the size of uptake $y . \mu_{\text {is }}$ is a dummy variable in which the value 1 is assigned to an uptake measure $i$ from a study $s$ in which enrolment was opt-out and 0 if it was opt-in. This is included in all specifications because the research on opt-in versus opt-out enrolment suggests that opt-out enrolment rates are substantially different to opt-in rates.

To estimate the relative contribution that each covariate makes to explaining the variation in $\gamma$, each covariate represented by $\chi_{i s}$ in the equation above is introduced separately, in independent regression analyses in which $\chi_{i s}$ is respectively; a dummy variable or a series of dummy variables indicating whether the uptake measure $i$ from a study $s$ run in Great Britain, the Netherlands, Australia, Norway or France, in which the United States is the omitted dummy and therefore the reference category; a series of dummy variables indicating whether the uptake measure $i$ from study $s$ relates to a capacity pricing ${ }^{8}$ tariff, a critical peak rebate tariff, a dynamic TOU tariff, real-time pricing tariff, static TOU tariff combined with critical peak pricing, a static tariff combined with real time pricing, an inverse static TOU tariff (in which the peak rate is overnight rather than during the day), a static TOU tariff plus a static TOU tariff combined with critical peak pricing and a static TOU tariff plus a static TOU combined with critical peak pricing and a critical peak rebate, ${ }^{9}$ in which a static TOU tariff (in which the

\footnotetext{
${ }^{7}$ OLS regression was selected because parametric tests such as OLS have greater statistical power than non parametric tests and, despite the small sample size small, kernel density plots show that the data does not deviate substantially from a normal distribution and econometric textbooks find that OLS generates unbiased and efficient results even when the underlying distribution is not perfectly normal.

${ }^{8}$ Capacity pricing tariffs charge customers for the demand in kilowatts (kw) rather than consumption in kilowatt hours (kWh) such that the customers' bill is determined by the maximum amount of electricity used at any point during the billing period. Although capacity pricing tariffs are therefore different from the other types of TOU tariffs discussed in this paper, they are included here where they form part of wider TOU trials - but they were note explicitly sought. Their inclusion is merited because such tariffs aim to achieve some of the aims of demand-response, namely to minimise unpredictable peaks in electricity demand during any billing period.

${ }^{9}$ The penultimate two categories contain multiple tariffs because the study from which the measure of uptake was drawn enrolled participants into a trial in which they would have been randomly assigned to different types of tariffs. As such, the measure of uptake cannot be disaggregated by tariff type but instead arguably reflects a persons' willingness to participate in a trial in which
} 
peak rate is during the day rather than overnight) is the omitted dummy variable and therefore the reference category against which the coefficient $\beta$ on each covariate should be compared; a dummy variable indicating whether the uptake measure $i$ was from a study $s$ in which the tariff was framed to potential consumers as being able to save them money (a money frame), and zero otherwise, excluding studies in which it was not possible to identify what framing was used; a dummy variable indicating whether the uptake measure $i$ was from a study $s$ in which the tariff was framed to potential consumers as being able to save them money and help the environment (an environmental frame), and zero otherwise, excluding studies in which it was not possible to identify what framing was used; a dummy variable in which the value 1 is assigned to a measure of uptake $i$ in study $s$ in which the tariff was accompanied by bill protection and zero otherwise; a dummy variable in which the value 1 is assigned to a measure of uptake $i$ in study $s$ in which participants were offered an upfront cash payment and zero otherwise, excluding uptake measures from all survey experiments ${ }^{10}$; a dummy variable in which the value 1 is assigned to a measure of uptake $i$ in study $s$ in which the tariff was accompanied by an automation device that allows consumers to remotely adjust their electrical devices in response to the price or which allows a third party, usually the supplier, to do so on their behalf. The term $\delta_{\mathrm{s}}$ is a fixed effect for each study $s$ from which the measure of uptake $i$ was taken, implemented as a series of dummy variables for each study.

Fixed effects are included because Ordinary Least Squares regression assumes that uptake measures are identically and independently distributed across studies. However, this is unlikely to be true because many uptake measures are recorded from the same studies, and uptake measures from the same study are likely to be correlated because they are based on the same population, same tariffs and so on. One option is to cluster standard errors at the study level, however this only controls for the average correlation in uptake measures within studies rather than specific intra-cluster correlation for each study. Including fixed effects for each study adjusts standard error estimates for specific intracluster correlation. ${ }^{11}$ Studies did not report uptake by different population sub-groups to enable meaningful analysis of heterogeneity across factors such as age and income. The alpha value is set at the conventional level of $\mathrm{p}<0.05$. However, we also interpret results at lower confidence levels, using confidence intervals, to assess whether it is more likely than not that a particular factor is correlated with uptake. ${ }^{12}$

\section{Results}

\subsection{Results of the search}

Fig. 1 describes how the references identified through the searches

(footnote continued)

they could be enrolled on any of the tariffs.

${ }^{10}$ Cash incentives are usually provided to compensate people for the inconvenience of participating in a trial or to attract consumers to participate in a trial or to sign up to a tariff; in surveys, cash is used as payment for undertaking the survey so it does not serve the same purpose and would not be appropriate to consider it as such.

${ }^{11}$ An alternative method for accounting for nested data is to use the random effects estimator however study effects are unlikely to be random because studies were not randomly sampled from a larger population of studies. On the contrary, the systematized review design used means that studies were purposively sampled on the basis that they met specific inclusion criteria.

12 This is justified on the basis that the p-value is a continuous variable which, if interpreted as such, can provide a more informative or nuanced account of the likelihood that any given result is true. This is because, "In the same way that a small $\mathrm{P}$ value does not guarantee that there is a real effect, a $\mathrm{P}$ value just above 0.05 does not mean no effect."[57, p. 1], particularly if the independent variable of interest has a low prevalence in the population or has a small effect on the dependent variable of interest given the sample size.

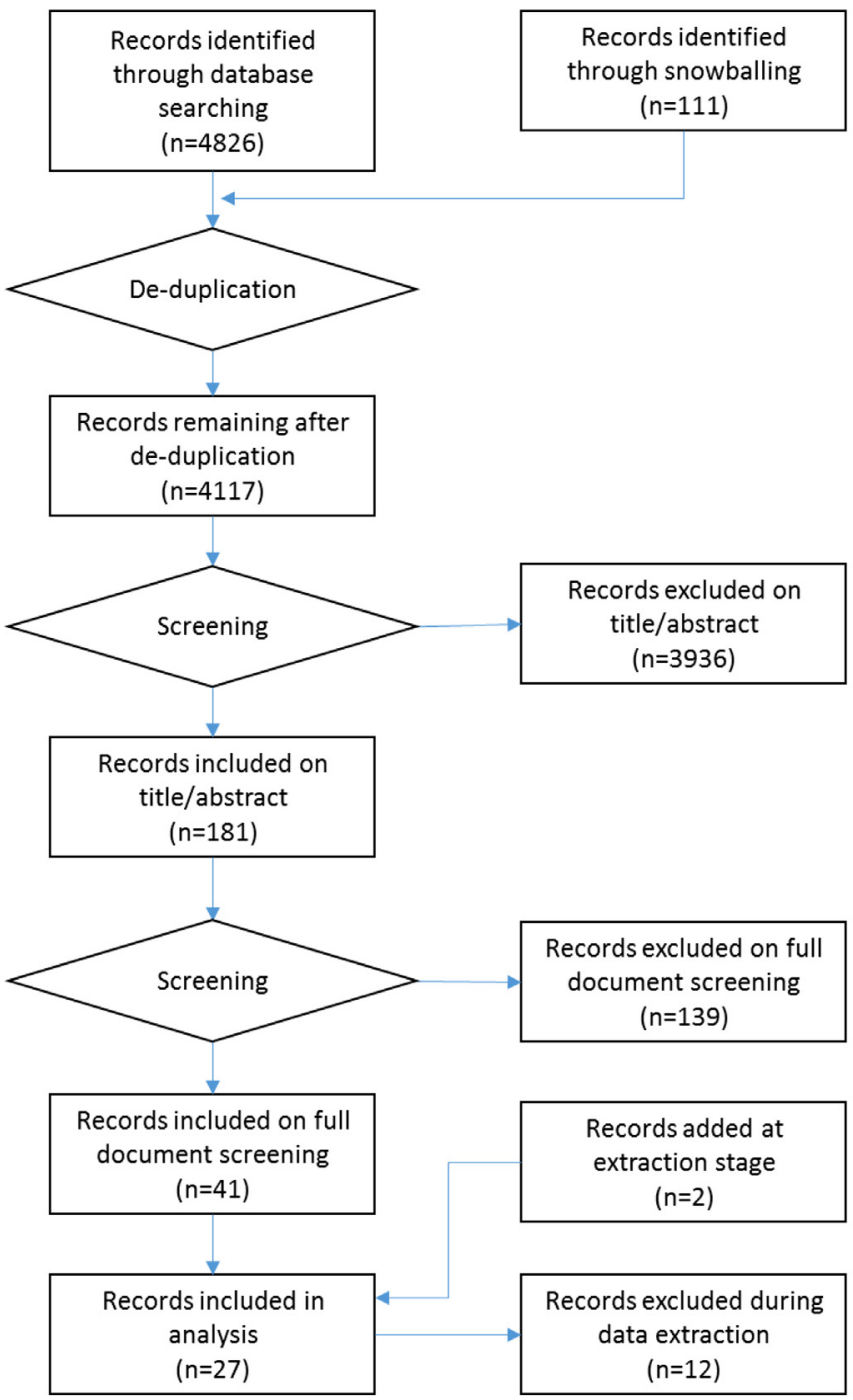

Fig. 1. PRISMA flow diagram of review process.

were processed for this review. A total of 41 documents were marked for inclusion. During extraction, two subsequent studies [21,22] were added that were not already included because the authors recognized their absence and knew that they met the screening criteria, and 13 studies were excluded because they either did not report a measure of uptake or because insufficient information was provided to compute a measure of uptake. During synthesis, a further three studies [18,21,23] were excluded because the sources did not provide information on the distribution of responses across the Likert scale measure of uptake to compute the proportion of switchers. This left a total of 27 studies for analysis covering 66 individual measures of uptake to a TOU tariff. ${ }^{13}$

\subsection{Characteristics of included studies}

Fig. 2 presents a heat map of the key characteristics of the studies included in which the size of the square represents the number of measures corresponding to the level of each factor. As can be seen, the majority of the evidence on consumer demand for TOU tariffs is from the United States; of the 66 measures of uptake included for analysis, 25 are from the United States, 15 from Australia, 15 from Great Britain, 8 from the Netherlands, 2 from France and 1 from Norway. Apart from

\footnotetext{
${ }^{13}$ Some studies ran multiple trial arms so provide multiple methods of uptake.
} 


\begin{tabular}{|c|c|c|c|}
\hline Factor & Level & & \\
\hline Study type & Survey & E & 34 \\
\hline & Trial recruitment & E & 20 \\
\hline & Commercial uptake & 口 & 12 \\
\hline Country & US & $\square$ & 25 \\
\hline & UK & 口 & 15 \\
\hline & Australia & 口 & 15 \\
\hline & Netherlands & ! & 8 \\
\hline & France & $=$ & 2 \\
\hline & Norway & - & 1 \\
\hline Tariff type & Static & $\square$ & 19 \\
\hline & CPP & a & 10 \\
\hline & RTP & a & 10 \\
\hline & Static + CPP & घ & 5 \\
\hline & CPR & = & 5 \\
\hline & DP & $=$ & 5 \\
\hline & CAP & a & 3 \\
\hline & Static + CPP + CPR & . & 2 \\
\hline & Static + RTP & - & 1 \\
\hline & Static inverse & $\cdot$ & 1 \\
\hline Automation & Yes & 口 & 15 \\
\hline & No & & 37 \\
\hline & Unknown & घ & 7 \\
\hline Benefit frame & Money & & 48 \\
\hline & Money \& environment & - & 2 \\
\hline & Unknown & = & 8 \\
\hline Default frame & Opt-in & & 62 \\
\hline & Opt-out & m & 3 \\
\hline Bill protection & Yes & 뭄 & 12 \\
\hline & No & & 49 \\
\hline & Unknown & = & 5 \\
\hline Additional & Yes & 目 & 9 \\
\hline incentive & No & $\square$ & 19 \\
\hline & Unknown & " & 4 \\
\hline
\end{tabular}

Fig. 2. Characteristics of the studies included in the review.

Great Britain and the United States, the evidence on all countries comes from a single study.

The majority of the evidence relates to uptake to static TOU tariffs and is based on stated willingness to switch to tariffs, as measured amongst participants in specially commissioned surveys, as opposed to uptake rates to commercially available tariffs or the proportion of participants who agreed to go onto a TOU tariff as part of their participation in an academic trial. Of the 12 measures that are based on the proportion of consumers signing up to a commercially available TOU, nine are from the United States, two are from France (EDF Tempo, EDF TOU) and one is from GB (Economy 7).

Most of the measures of uptake are based on opt-in rather than optout recruitment methods, and very few used bill protection or an additional participant financial incentive to encourage uptake. The predominant way in which TOU tariffs in the sample were framed to consumers is to emphasise that TOU tariffs can save money, and just a 
Table 3

Average uptake to TOU tariffs by study design, country, tariff design, default frame, benefit frame, bill protection, additional financial incentive and automation.

\begin{tabular}{|c|c|c|c|c|c|}
\hline Factor and level & Mean (\%) & Median (\%) & $\begin{array}{l}\text { Lower 95\% } \\
\text { confidence } \\
\text { interval }(\%)\end{array}$ & $\begin{array}{l}\text { Upper 95\% } \\
\text { confidence } \\
\text { interval (\%) }\end{array}$ & $\mathrm{N}$ \\
\hline \multicolumn{6}{|l|}{ Study type: } \\
\hline Survey & 37 & 36 & 31 & 43 & 34 \\
\hline Trial recruitment & 23 & 12 & 10 & 36 & 20 \\
\hline $\begin{array}{l}\text { Commercial sign } \\
\text { up }\end{array}$ & 17 & 7 & 1 & 33 & 12 \\
\hline \multicolumn{6}{|l|}{ Country: } \\
\hline Australia & 51 & 54 & 46 & 56 & 15 \\
\hline UK & 30 & 30 & 22 & 37 & 15 \\
\hline US & 25 & 9 & 10 & 35 & 25 \\
\hline Norway & 25 & 25 & - & - & 1 \\
\hline France & 19 & 19 & - & - & 2 \\
\hline Netherlands & 14 & 13 & 4 & 25 & 8 \\
\hline $\begin{array}{l}\text { Static }+ \text { (Static } \\
+ \text { CPP) } \\
+ \text { CPR }\end{array}$ & 44 & 44 & - & - & 2 \\
\hline CPR & 53 & 53 & 14 & 70 & 5 \\
\hline CAP & 39 & 37 & 23 & 36 & 3 \\
\hline Static & 35 & 35 & 24 & 46 & 22 \\
\hline $\mathrm{CPP}$ & 28 & 21 & 12 & 43 & 10 \\
\hline $\mathrm{DP}$ & 22 & 25 & 8 & 36 & 5 \\
\hline RTP & 17 & 5 & 2 & 33 & 10 \\
\hline Static + RTP & 12 & 12 & - & - & 1 \\
\hline Static + CPP & 9 & 6 & - & - & 4 \\
\hline $\begin{array}{c}\text { Static }+ \text { (Static } \\
+ \text { CPP) }\end{array}$ & \multicolumn{4}{|c|}{+ (РP) } & 1 \\
\hline $\begin{array}{l}\text { Static inverse } \\
\text { Default frame: }\end{array}$ & 1 & 1 & - & - & 1 \\
\hline Opt-out & 83 & 87 & 57 & 108 & 3 \\
\hline Opt-in & 26 & 25 & 21 & 32 & 62 \\
\hline \multicolumn{6}{|l|}{ Benefit frame: } \\
\hline \multicolumn{6}{|l|}{ Environment } \\
\hline Money & 30 & 23 & 22 & 37 & 48 \\
\hline Unknown & 26 & 26 & 14 & 38 & 16 \\
\hline \multicolumn{6}{|l|}{ Bill protection } \\
\hline Yes & 35 & 32 & 16 & 53 & 12 \\
\hline No & 27 & 28 & 21 & 33 & 49 \\
\hline Unknown & 35 & 25 & - & - & 5 \\
\hline \multicolumn{6}{|l|}{$\begin{array}{l}\text { Additional } \\
\text { financial } \\
\text { incentive: }\end{array}$} \\
\hline Yes & 35 & 36 & 28 & 41 & 35 \\
\hline No & 20 & 12 & 10 & 30 & 27 \\
\hline Unknown & 37 & 26 & - & - & 4 \\
\hline \multicolumn{6}{|l|}{ Automation: } \\
\hline Yes & 31 & 28 & 16 & 46 & 15 \\
\hline No & 32 & 33 & 25 & 39 & 33 \\
\hline Unknown & 18 & 7 & 4 & 31 & 7 \\
\hline
\end{tabular}

Note: Due to small sample sizes it was not possible to compute confidence intervals for all the variables recorded; these cells are marked with a dash to indicate that they are intentionally left blank.

small number of measures $(n=2)$ are also drawn from studies which emphasised the environmental benefits. No other ways of framing the tariffs were used.

\subsection{Risk of bias in included studies?}

The Cochrane Collaboration's 'Risk of Bias' tool suggests that bias be considered along five domains - selection, performance, attrition, detection, reporting - and an 'other bias' category to capture threats to internal validity. Risk of bias was not assessed during the review but is being assessed here. Detection bias is a problem in this review insofar as that the review only included reports written in English, so our figures may be more representative of English speaking OECD countries, which is a relatively minority of the 35 OECD countries. Screening of webpages was only performed for UK organisations which could also lead to detection bias.

\subsection{Results of individual studies - overall consumer demand for TOU tariffs and other relevant factors}

The variation in mean uptake to TOU tariffs is large, ranging from a mean of $0-96 \%$. The mean enrolment rate is $29 \%$ with a standard deviation almost as large $(\mathrm{sd}=24 \%)$ and the median enrolment rate is $27 \%$. The variation in uptake across studies may be explained by a number of factors, including study type, country and tariff design, as identified by the research questions.

Table 3 presents a breakdown of the mean and median ${ }^{14}$ uptake according to these factors, sorted in descending order of the mean (with the exception of the yes/no/unknown questions), with the lower and upper $95 \%$ confidence intervals also presented. For some measures, uptake is taken from studies in which participants were randomly assigned to one of two TOU tariffs (e.g. static TOU or static TOU combined with critical peak pricing) so uptake cannot be disaggregated by tariff type and is therefore presented as uptake for two or more tariff types.

The most notable differences in uptake are those between study type and recruitment method, whether opt-in versus opt-out, a relationship which is made clearer in Fig. 3, which is a bar chart of the mean uptake to any TOU tariff for each of the 66 measures of uptake obtained across the 27 studies. With just one exception [24], the highest measures of uptake are recorded from studies using opt-out recruitment [25-27] and studies using willingness to switch as a proxy for potential uptake $[15,22,28-30]$. Although the mean uptake for commercial tariffs is lower in magnitude than recruitment rates for trials the difference is not statistically significant $(\mathrm{p}=0.490)$ which is why these are grouped together with measures from commercial tariffs.

As a result, all analyses include controls for whether the study is a survey and whether enrolment is opt-out, with the results presented in Table 3. The coefficients in Table 3 are a product of a series of linear regressions in which uptake is the dependent variable measured as a proportion from 0.0 to 1.00 and different factors are the independent variables. Since an analysis of variance test reveals that the intra-cluster correlation is 0.47 which is high and demonstrates that it is not appropriate to assume that the error term is independently distributed, fixed effects are used in nearly all analyses. Note that, study fixed effects will also penalise results that are strongly dependent on results from a single or very few studies and which may therefore be less reliable than results from multiple studies (however, conversely, the fixed effects could also mask effects that are constant across studies, which may therefore be reliable results, which is why we use and interpret results which include these effects carefully).

Throughout columns (1) to (9) in which a range of control variables are added, uptake measures elicited from surveys are consistently estimated as being between $28 \%$ and $36 \%$ points higher than uptake to commercially available TOU tariffs or tariffs people were able to sign up to in trials, after controlling for intra-cluster correlation between measures obtained from the same surveys using fixed effects. Opt-out enrolment is estimated as being consistently $70 \%$ points higher, after controlling for intra-cluster correlation. When both measures are inputted into the regression analysis, the model estimates that they explain $85 \%$ of the variation in uptake to TOU tariffs (column 1, Table 4).

There are significant differences in uptake between countries, but when study type is controlled for the only significant remaining difference is between the UK and Australia ( $<<0.05)$. As the Australian evidence is drawn from a single study, this is most likely due to specific

\footnotetext{
14 The median is also presented given that, for some variables, the distribution is highly skewed due to the large variation in uptake measures.
} 


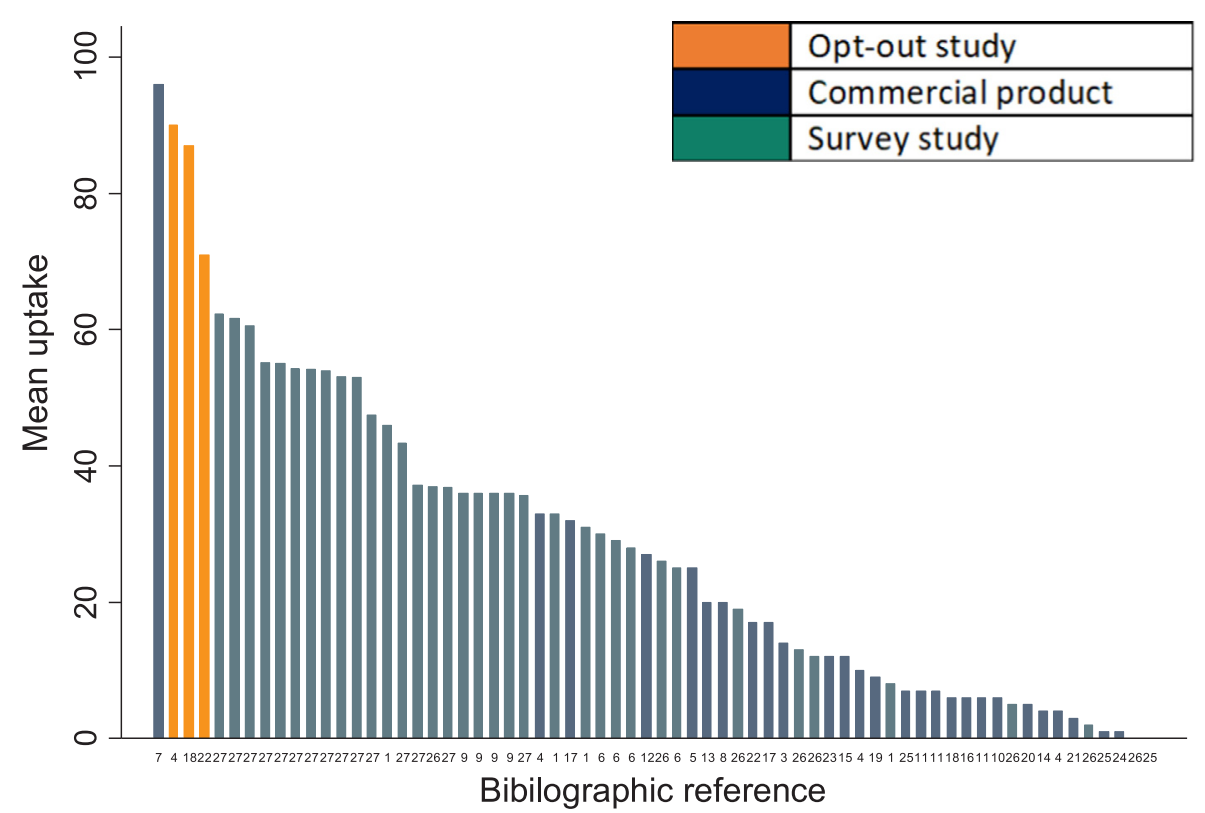

Fig. 3. Consumer measures of demand for TOU tariffs by study type and default frame. Note: Each bar represents a measure of uptake. Some studies obtained multiple measures so individual studies may appear multiple times. All studies used opt-in enrolment unless they are highlighted as having used opt-out. The horizontal axis provides the bibliographic reference for each study (see Table A.1 in the appendix for the references). design considerations of this individual study.

There appears to be substantial variation in average uptake depending on tariff design in the raw data (Table 2). The regression model estimates that real time pricing tariffs are correlated with a $13 \%$ lower level of uptake when compared to a static TOU tariff ( $p<0.01$ ), when controlling for recruitment method, whether uptake was measured in a survey and for correlation in uptake measures recorded within the same studies (column 3). The most highly specified model (column 3) also estimates that static TOU tariffs are preferred to capacity pricing $(\mathrm{p}<0.001)$ and marginally statistically significantly more popular than dynamic pricing tariffs $(\mathrm{p}<0.10)$. No other differences are statistically significant. This is possibly due to the low sample size for these other tariff designs. When regression analysis is run with only the different tariff designs as control variables (column 4), the adjusted RSquared value indicates that tariff design explains $9 \%$ of the variation in uptake, which is lower than for the other factors considered so far.

There is no statistically significant difference in uptake across measures of uptake obtained from studies in which people were told about the potential financial savings from switching tariff $(p=1.000)$ or the financial and environmental benefits $(p=1.000)$.

Offering people bill protection $(\mathrm{p}=0.184)$ does not appear to have a statistically significant impact on uptake (column 7), however it does once removing the fixed effects which control for correlation in uptake across studies $(\mathrm{p}<0.05)$ which is likely to be reflective of the fact that only very few studies tested bill protection and all had relatively high levels of uptake (for brevity, results not reported in Table 3).

Providing people with upfront financial payments for signing up to a TOU tariff either in trials or for commercial offerings (survey measures excluded) has a strong statistically significant positive effect on uptake ( $p<0.001)$ regardless of whether the specification controls for correlation in uptake within studies $(\mathrm{p}<0.01)$.

Some TOU tariffs are accompanied by automation devices (usually smart thermostats which customers can use to remotely control their space heating and cooling e.g. to avoid peak times) but the data suggests that uptake is not related to the presence of automation $(p=0.958)$, even after removing controls for intra-cluster correlation $(\mathrm{p}=0.158) .{ }^{15}$ In further exploratory analyses, not reported in Table 3 , automation was also not found to have any effect on uptake to real time

\footnotetext{
${ }^{15}$ For brevity, the result without fixed effects is not reported in Table 3Error! Reference source not found.
}

pricing tariffs or dynamic tariffs $(\mathrm{p}=0.502)$ or real-time pricing tariffs on their own $(\mathrm{p}=0.299)$.

\section{Discussion}

\subsection{Demand for TOU tariffs}

Median uptake to a TOU tariff across 66 individual measures of uptake and 27 unique studies is $27 \%$. However, the variation in uptake is huge - the standard deviation around the mean is almost as large as the mean itself (24\%), with a range in uptake measures of 0-96\%. The results in Table 3 suggest this is most likely to be driven by variation in the way in which uptake is measured in studies as well as whether people were recruited to the tariff by default or via opt-in enrolment. Notably, the median proportion of domestic energy bill payers who say they would be willing to switch to a TOU tariff in national surveys is five times higher as the median enrolment rate to commercially available TOU tariffs. The higher uptake rate in survey research is consistent with the theoretical literature on the gap between behavioural intentions and behavioural action from psychology [31] and stated and revealed preferences in economics [32]. Together, method of measurement and whether recruitment is opt-out explain $85 \%$ of the variation in uptake to a TOU tariff.

We therefore recommend that demand for TOU tariffs is expressed as a range, based on the minimum and maximum mean recorded uptake and expressed for opt-in and opt-out enrolment separately. Survey measures could most usefully be viewed as indications of theoretical potential, which in reality are likely to be highly optimistic unless concerted action is taken to boost uptake.

Based on the available evidence, uptake to TOU tariffs is most likely to fall between $1 \%$ (the lower bound estimate for mean uptake to commercially available tariffs, most of which are offered in the US) and $43 \%$ (the upper bound estimate for mean willingness to switch obtained from surveys, most of which were run on nationally representative samples of British energy bill payers), depending on how much effort is made to engage consumers with TOU tariffs to close the gap between behavioural intentions and behavioural action [31-34]. If opt-out enrolment is used, uptake is most likely to exceed $57 \%$ but with an uncertain upper limit of enrolment approaching $100 \%$. It is not possible to say whether uptake is likely to vary across countries, for the reasons set out above.

Although the estimated range of TOU adoption rates under an opt-in 


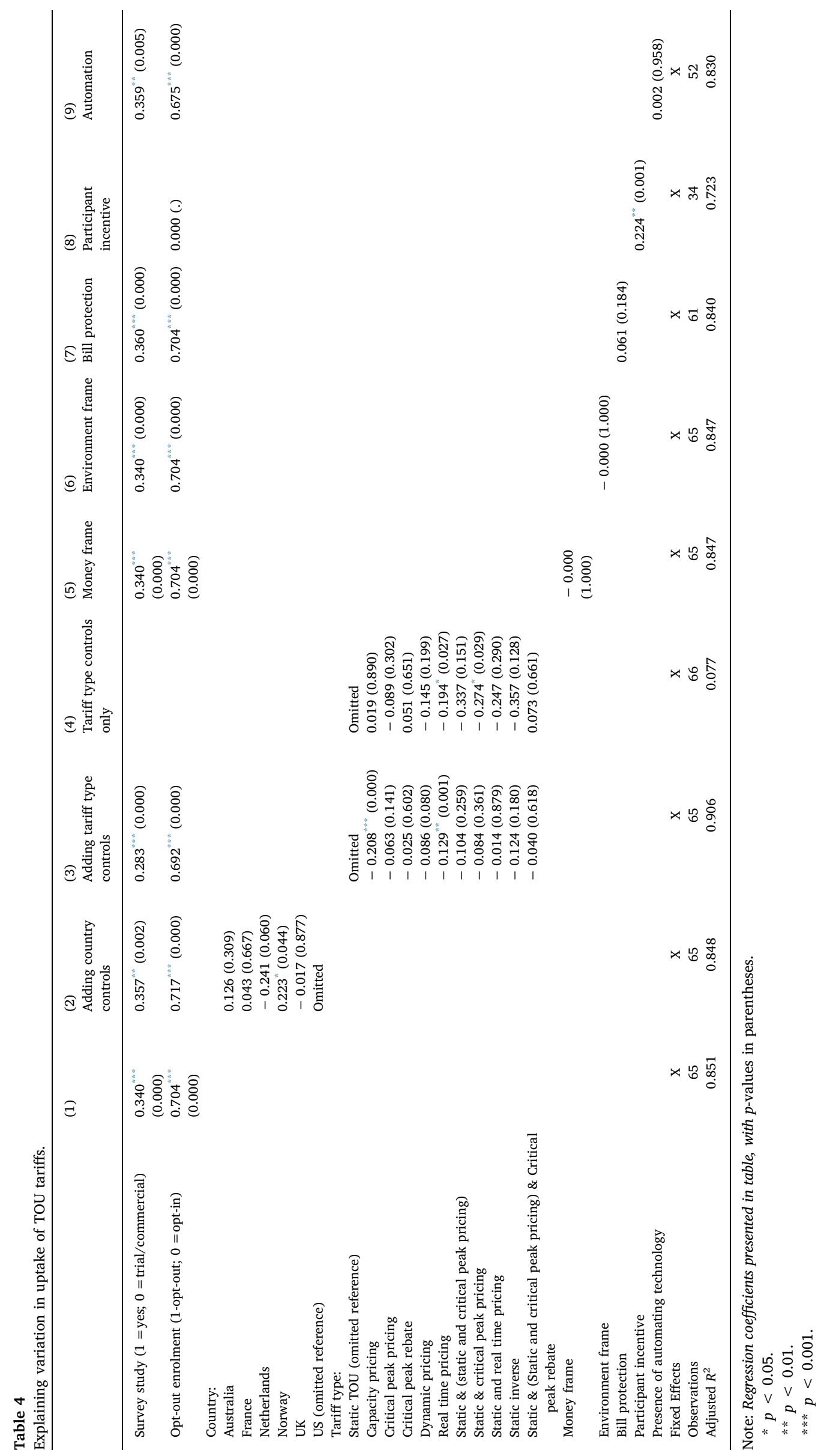


recruitment system is very wide, evidence from the literature on stated and revealed preferences [31-34] and the real-world behavior of consumers in the energy market [35] suggests that there is a strong risk that uptake is more likely to fall at the lower rather than higher end of the range presented above. Since the upper bound estimate of $43 \%$ comes from surveys measuring how willing people are to adopt a TOU tariff, it is best interpreted as capturing the maximum potential national uptake of TOU tariffs if every consumer who is willing to sign up to a TOU tariff at the time of the survey does indeed go on to sign up. However, since it is well known that behavioural intentions are a relatively poor predictor of future behavioural action, ${ }^{16}$ it also follows that a $40 \%$ adoption rate is unlikely to be achieved in reality unless substantial efforts are expended on encouraging switching. Consumer inertia is a major problem around the world with the majority of consumers having never left their home supplier since privatisation of retail electricity markets began over two decades ago [35].

At the same time, we also acknowledge that it is not possible to conclude with certainty whether observed differences in uptake between countries are due to genuine inter-country differences in consumer demand for TOUs (i.e. that consumers in GB and Australia are more in favour of TOU tariffs than consumers in the US) or due to differences in measurement method. Differences in uptake measurement method also overlap almost exactly with differences in the types of populations sampled. Survey recruitment has mostly been nationally representative $[15,28,29]$ whereas participants solicited to take part in TOU trials are, in many cases, very different to the average energy bill payer. ${ }^{17}$ Also, unlike for country, our model does not include separate controls for differences in recruited populations because, in most cases, non-nationally representative participant solicitation overlaps exactly with measurement method itself. However, on balance, our judgement is that it is highly unlikely that all of the variation in uptake across surveys and commercially available tariffs is attributable to crosscountry variation in demand for TOU tariffs and is more likely to be attributable to the fact that, for a variety of reasons, people are more likely to express an intention to switch to a TOU tariff than they are to switch to one in reality.

\subsection{The impact of tariff design}

As noted in [36], "different approaches to DSR can achieve different benefits for the network. While static tariffs allow regular peaks to be managed, they cannot incentivize demand that follows variable supply (such as from wind generation) or a response to unexpected peaks or faults. Dynamic tariffs, on the other hand, do permit such flexibility and are therefore potentially more valuable to networks" [36], p. 80. It is therefore important to understand whether there may be a gap between what tariffs consumers are attracted to and tariffs that are most useful for the electricity network.

The results suggest that real-time pricing tariffs, in which the price of electricity can vary freely throughout the day according to real-time supply and demand of electricity, are less popular than a static TOU tariff, in which the price bands apply for fixed periods each day or season. According to the results in Table 3, uptake to real-time pricing

\footnotetext{
${ }^{16}$ Unfortunately empirical studies testing the relationship between intentions and behavioural action do not provide a clear picture of the strength of the correlation between these two variables [34]. One meta-analysis finds that intentions explain $28 \%$ of the variation in behavioural action [31] whereas another reports frequency weighted average correlation between these two variables as 0.53 , with a lower $95 \%$ confidence limit of 0.15 and an upper limit of 0.92 [33].

17 For example, in cases where the overwhelming majority of people solicited to participate have central air conditioning $[24,57]$ or in cases where recruitment to a TOU tariff was undertaken amongst a pool of people who had already consented to have a smart meter installed as part of their participation in an earlier wave of the project [40].
}

tariffs is $13 \%$ lower ( $p<0.01$ ) than uptake to static TOU tariffs regardless of whether recruitment is opt-in or opt-out and whether uptake is hypothetical (measured in a survey) or actual (measured as trial recruitment or uptake to a commercially available real time pricing tariff). This result is robust to several specifications, including a specification including fixed effects to control for potential correlation in measures of uptake to real-time pricing tariffs that were obtained in the same studies.

Dynamic TOU tariffs, in which the price of electricity varies, usually within fixed parameters, freely throughout the day, and capacity pricing tariffs, are less popular than static TOU tariffs. The finding that capacity pricing might be less popular amongst consumers than static TOU is a relatively new one. However, the finding that real-time pricing tariffs and dynamic tariffs are less popular is broadly consistent with the results of individual studies which have systematically tested the impact of tariff design on uptake [15,17], including a study which was excluded from the meta-analysis because the outcome measure could not be standardised with those of other studies [18]. This aversion to real time pricing tariffs may be due to their unpredictability and greater complexity, for example, one study considered here which systematically tested the impact of tariff design on uptake found that dynamic TOU tariffs were rated consistently lower than static TOU tariffs for perceived ease of use and control, even though overall willingness to switch did not vary [15].

Although a number of other types of TOUs were captured during the review, the results do not point to any consistent statistically significant differences between static TOU tariffs and alternative designs such as critical peak pricing, critical peak rebates and static TOU layered with critical peak pricing and/or rebates. Compared to static TOU tariffs, however, these tariff designs were tested much less frequently, suggesting that the sample size is not large enough to robustly test for differences. This means that such tariffs may be more or less popular than static TOU tariffs but that our data was not able to pick this up; or, rather, the public have had insufficient exposure to these alternative tariff designs to judge how they are likely to be perceived. Until further evidence emerges, it is recommended that policymakers and researchers assume that there will be no difference in uptake to critical peak rebate programmes as compared to static TOU tariffs.

When it comes to critical peak pricing tariffs, policymakers may wish to be guided by the results of three survey experiments which found that significantly fewer people were willing to switch to a critical peak pricing tariff as compared to a static time of use tariff [28] and a flat-rate tariff $[17,23]$.

Finally, and perhaps more importantly, given that consumers are likely to always have the choice between flat-rate tariffs and TOU tariffs, it is worth noting that in studies that have directly compared uptake to flat-rate tariffs and TOUs, uptake to the flat-rate tariff has been significantly higher $[17,18]$.

\subsection{Recruitment strategies to increase uptake to TOU tariffs}

Given the low lower bound estimate of demand for TOU tariffs (1\%) - and the relative unpopularity of real-time pricing tariffs, which offer greater potential benefits to the electricity system - it is important to consider how uptake might be increased if the full benefits of TOUs are to be realised. To get a sense for how tariff uptake might be increased, meta-regression analysis was undertaken to help to obtain answers to the following questions:

1. What is the correlation between uptake and making enrolment optout rather than opt-in?

2. What is the correlation between uptake and bill protection?

3. What is the correlation between uptake and automation?

4. What is the correlation between uptake and offering upfront cash payment for signing up?

5. What is the correlation between uptake and telling people about 
financial savings from switching to a TOU tariff?

6. What is the correlation between uptake and other types of message framing, other than financial messaging?

The studies provide strong evidence that opt-out enrolment increases uptake because of the three trials which tested this approach, two of them used a randomised control trial design with very large sample sizes and robust designs [26,37]. Median enrolment rates under opt-out policies are nearly four times higher than uptake under an optin approach. The outlying 96\% uptake in an opt-in study [24] is for a US programme in which most customers had central air conditioning that the programme allowed them to put on a timer to help avoid the peak prices. The paper reporting these findings states that "the high sign-up rate is directly attributed to an intense marketing effort consisting of: (1) an initial mailing to introduce the $\mathrm{CCC}^{\mathrm{TM}}$ programme; (2) follow- up phone calls and door drop-offs to arrange an in-person meeting; and (3) subsequent workshops to answer questions." [24], p. 899. However, a number of other trials [38-40] used similar recruitment methods and did not achieve these high enrolment rates so it is hard to explain why this programme was so successful and suggests it is best treated as an anomaly. However, as argued elsewhere [41], opt-out enrolment may not be the most appropriate recruitment strategy for TOU tariffs. For example, there may be public acceptability concerns around automatically switching people onto TOU tariffs, even if they can opt-out because people who do not realise they have been switched could face higher costs if they do not shift their electricity usage.

The studies provide some indication of the answers to \#2- \#4, once also considering the results of individual survey studies that manipulated the variables of interest experimentally. Providing small upfront financial payments (e.g. shopping vouchers [42] or small cash payments) is estimated to increase uptake by $22 \%$ in the meta-regression. However, we cannot confidently attribute the differences in uptake to this cash incentive rather than other differences between studies that do and do not use cash incentives because none of the studies compare uptake to a tariff when a cash incentive is offered to a control group that was not offered a cash incentive.

The meta-regression revealed no statistically significant difference in uptake across studies in which the tariff was offered with bill protection or automation however we do not have enough data to be highly confident that this means that bill protection and automation have no effect on uptake or whether too few studies offered these features to provide sufficient power to detect an effect or because the impact is being masked by other confounding variables given that very few studies manipulated these factors experimentally. This is likely to be because, as mentioned above, the focus on the literature so far has been on whether tariffs change people's consumption patterns rather than whether or why people would sign up to such a tariff of their own accord in the first place.

Nevertheless, studies $[17,18]$ which explicitly tested the impact of automation on uptake do find a statistically significant increase in uptake, with one study finding it increased willingness to switch by $4.5 \%$ [17]. One study reported interaction effects between automation and tariff type finding that automation made a dynamic TOU tariff as popular as a static TOU tariff but that automation made no difference to the attractiveness of the static tariff [15]. The only study to explicitly test the impact of bill protection on uptake, in which survey participants were randomly assigned to a number of TOU tariffs either with or without bill protection, found that bill protection increased willingness to switch to a TOU tariff by $10 \%$ [17].

Questions \#5-\#6 are substantially harder to answer using existing data because nearly all studies, apart from one [29], either tell people the tariff will save them money (a money frame) or do not specify what frame was used at all. Two studies excluded from this review because it was not possible to standardise their uptake measures [21,23] - in addition to the study which was included [29] - found mixed results as to the impact of telling consumers about the environmental benefits of TOU tariffs on uptake. Since nearly all energy tariff marketing already frames switching tariff as a way of saving money, there is no evidence as to whether changing this approach could increase uptake to TOU tariffs, which, are likely to provide much lower savings than just switching to the cheapest available flat-rate tariff.

\subsection{Key limitations of the method}

The review was as comprehensive as possible given the resources available. However, it is possible that either the selection of search terms or locations (e.g. some additional UK and US organisations were scanned but not other countries) meant that certain documents were missed. However, we assess the risk of having missed any major projects (which would have substantially affected the results) as low.

The key protocol variation that may have been significant for the findings is that we designed the search to locate studies which specifically set out to measure TOU uptake, but later expanded this to include recruitment metrics into larger TOU studies where the primary aim was to measure demand response. While we believe that our search strategy is likely to have identified reports of such studies which provided sufficient information on recruitment, it is possible that informative studies may have been missed. However, there is no reason to believe that the reasons for study inclusion would have led to systematic under- or over-estimation of uptake.

\section{Conclusions and policy and research implications}

\subsection{Key findings}

The key contribution of this systematized review is to synthesise a wide range of evidence on demand for TOU tariffs from over 27 studies, incorporating 66 individual measures of uptake to various TOUs in different countries, using different recruitment methods and measured in different ways. The aim of the review was to identify the likely uptake of TOUs amongst domestic energy consumers, and what factors influence uptake.

The results indicate that uptake to TOU tariffs is most likely to fall between $1 \%$ and $43 \%$, depending on how much effort is made to engage consumers with TOU tariffs to overcome customer inertia and thereby close the gap between behavioural intentions and behavioural action. Since consumers rarely switch supplier, there is a high risk that opt-in enrolment rates could fall substantially below $43 \%$ unless effective recruitment methods are found. We therefore strongly suggest that all effort is made to encourage consumers to adopt TOU tariffs to avoid the risk of enrolment rates being closer to the lower bound confidence limit of $1 \%$. If people are recruited onto TOU tariffs by default, uptake is likely to exceed $57 \%$ and could approach $100 \%$.

The measure of uptake does not account for variation in uptake over time, and it is acknowledged that adoption of new tariffs is likely to be a gradual process. However, this is the best available indicative range of potential for future uptake to TOUs at this time. Since the majority of the studies that met the inclusion criteria were run in United States, Great Britain and Australia, it is recommended that policymakers apply caution when attempting to apply these results beyond these countries.

The results of the meta-analysis provides good evidence that realtime pricing tariffs and dynamic TOU tariffs are less preferred to static 
TOU tariffs. However, small sample sizes on other tariffs types means that it is not possible to conclude whether other tariff types (e.g. critical peak rebates, inverse TOU) will be more or less popular than static tariffs. There is some evidence from the results of individual studies $[17,23,28]$ to suggest that critical peak pricing tariffs will be less popular amongst consumers than TOU tariffs and flat-rate tariffs. The results suggest that automation and bill protection are more likely than not to increase uptake to TOU tariffs, but more research is required to provide greater confidence in these results.

\subsection{Next steps for research}

The wide range of uptake estimates described above means it is hard for governments to plan on certain levels of TOU penetration. More research is required to increase the precision of estimates and help ensure countries can make realistic plans, and avoid falling short of enrolment targets. For example, an enrolment rate of $1 \%$ (the lower bound estimate from the meta-analysis) could have a substantial impact on the UK Government's smart meter cost benefit analysis, which relies on uptake of $30 \%$ by 2030 [43]. More work is also needed to test the impact of non-static tariff designs on uptake to TOUs relative to static tariffs. Critical peak rebates especially have the advantage that they are non-punitive, in the sense that consumers can only ever be better off, but not worse off by participating, which is attractive from a policy perspective.

How can uptake estimates be made more precise? The review highlighted a large gulf between the results obtained from surveys and those obtained from studies in which people were able to switch to a TOU. Given the lack of evidence of uptake based on actual switching rates, it is strongly recommended that countries obtain a measure of uptake based on recruitment rates to commercially available nextgeneration TOUs. Where such commercial offerings are not available, innovative solutions will be required to undertake this research. In GB [44] and Ireland [45], for example, the energy regulators have plans to create a 'regulatory sandbox' that would permit suppliers to test new offerings amongst consumers prior to full launch. Alternative methods which do not require the participation of an energy supplier are to run trials in which the outcome measure is not switching itself but rather proxies for switching or behavioural antecedents to switching, such as willingness to engage with information about switching to a TOU. Surveys will still have a useful role to play. They permit rapid testing (under experimental conditions) of a wide range of tariff and marketing characteristics, helping to inform commercial product development.

With the exception of three survey experiments $[15,17,29]$, the studies considered in this review have not explicitly sought to test methods of increasing uptake to TOUs that do not rely on opt-out enrolment. This is problematic because it means that it is not possible to identify whether differences in design or marketing are causally related to uptake. Where opt-out enrolment is politically unacceptable (due to public concern and the risk of some people losing money [41]), policymakers may prefer to leave consumers to opt-in to TOU tariff rates. This review therefore highlights the need for experimental research on how to encourage people to switch from a flat-rate tariff to a TOU which aims to encourage self-selection of consumers best suited to DSR whilst eliminating the major potential disadvantages of both opt-in (sub-optimal uptake) and opt-out (passive, uninformed decision making) methods. To enable robust causal inferences, this research must involve the random assignment of participants to variations of the same tariff to test which variation results in higher switching rates as argued by other authors too [16]. For example, the review provides good evidence that small upfront cash payments are positively correlated with uptake of TOUs. Future research could test this experimentally and could consider varying the size, timing and reward type to test whether this has a causal impact on uptake.

The synthesis also indicates that automation and bill protection are two promising strategies. The advantage of automation is that it can also serve to increase potential customer savings, thus increasing the total pool of people who could save money on a TOU. Automation reduces, and bill protection removes, the possibility that people could end up paying more on a TOU than on their flat-rate tariff, and research suggests that fear of losing money (loss-aversion) is associated with a statistically significant reduction in willingness to switch to a TOU tariff [29]. However, more research is required to establish whether bill protection and/or automation does indeed have a causal impact on uptake to TOU tariffs with higher levels of precision and statistical confidence than was possible at this point.

In particular, the synthesis highlights a substantial unexploited opportunity to test the impact of different types of message-framing on uptake to TOUs. This is a very low-cost intervention that has had significant impacts in other domains including increasing registrations to the organ donor register [46], enrolment into university [47] and encouraging timely payment of taxes [48] and fines [49]. There are many different ways of framing messages, and the literature to date has so far only tested telling people about the financial savings from switching to a TOU. However, as this review suggests, the average savings are relatively modest which may explain why no evidence was found that monetary messages were associated with an increased uptake. Moreover, evidence from fields such as social psychology [50,51], behavioural economics [52] and sociology [53] suggests that people are motivated by a wider range of considerations than just money, as pointed out in another recent review article [54]. For example, messaging could include the use of social norms, or tailoring of offerings to target consumer groups with electric heating or vehicles.

Finally, the synthesis also reveals several shortcomings in the reporting of results, which future research could address. For example, this review identified studies that met all the inclusion criteria except for that they did not provide information on recruitment e.g. [55,56]; future trials and studies which seek to measure the impact of TOUs on demand profiles should report recruitment rates or at least provide information that permits the calculation of recruitment rates (the total number of participants solicited and the total number recruited). Trials should also report on energy bill impacts where possible. Regulators could consider inviting or requiring energy suppliers to inform them of uptake to their commercially available TOUs so that this information can be used to update government carbon strategies and smart meter cost-benefit analyses.

\section{Acknowledgements}

The authors gratefully acknowledge Citizens Advice (a consumer advice charity) for funding the review. Additional funding to prepare the work for academic publication was received from the RCUK Centre for Energy Epidemiology (grant number EP/K011839/1) and the EPSRC Centre for Doctoral Training in Energy Demand (LoLo) (grant numbers EP/L01517X/1 and EP/H009612/1). The authors would particularly like to thank Ryan Hledik for his feedback on the findings from this work.

\section{Competing interests}

The authors declare no competing interests. 


\section{Appendix A}

See Table A.1

Table A.1

Bibliographic references for all studies included in review numbered according to the references in Fig. 3 in the main text.

1 BEIS. Smart Energy Research - BEIS Consumer Panel. November 2016, 1-5. https://www.gov.uk/government/uploads/system/uploads/attachment_data/file/566230/Smart Energy_Consumer_Panel_Research_Summary_Report.pdf.

2 Phillips R, Owen G, Ward J. Project Lessons Learned from Trial Recruitment: Customer - Led Network Revolution Trials. July 2013 , 1-46.

3 IHS Global Insight. Demand Side Market Participation Report for Department of Energy and Climate Change. London, UK, 2009, 1-50. http://webarchive.nationalarchives.gov. uk/20121217150421/http://decc.gov.uk/assets/decc/Consultations/Electricity supply security/1_20090804144704_e_@ @_DSMreportGlobalInsight.pdf. Accessed July 7, 2015.

4 Charles River Associates. Primer on Demand-Side Management with an Emphasis on Price-Responsive Programmes - Report Prepared for The World Bank, February 2005, 1-71. http://www.worldbank.org.

Ericson T. Households' self-selection of dynamic electricity tariffs. Appl Energy. 2011; 88(7):2541-2547.

Fell MJ. Taking charge: perceived control and acceptability of domestic demand-side response. 2016. Unpublished thesis. http://discovery.ucl.ac.uk/1475103/.

Hartway R, Price S, Woo CK. Smart meter, customer choice and profitable time-of-use rate option. Energy. 1999; 24(10):895-903.

Schofield J, Carmichael R, Tindemans S, Woolf M, Bilton M, Strbac G. Residential Consumer Responsiveness to Time-Varying Pricing - Low Carbon London Learning Lab, Report A3 for the "Low Carbon London" LCNF project: Imperial College London, 2014, 1-77.

9 Nicolson M, Huebner G, Shipworth D. Are consumers willing to switch to time of use electricity tariffs? The importance of loss-aversion and electric vehicle ownership. Energy Res Soc Sci. 2017;23:82-96.

10 Schare, S. et al. NSTAR Smart Grid Pilot Final Technical Report, Navigant Consulting, February 2015, 1-67.

11 PEPCO, PowerCents DC Program Final Report, eMeter Corporation, September 2010, 1-78.

12 Qiu Y, Colson G, Wetzstein ME. Risk preference and adverse selection for participation in time-of-use electricity pricing programmes. Resour Energy Econ. 2017;47: 126-142.

13 Herter K. Residential implementation of critical-peak pricing of electricity. Energy Policy. 2007, 35:2121-2130.

14 Baladi SM, Herriges JA, Sweeney TJ. Residential response to voluntary time-of-use electricity rates. Resour Energy Econ. 1998, 20(3):225-244.

15 Neenan B, Patton M. FirstEnergy's Smart Grid Investment Grant Consumer Behavior Study Final Evaluation. June 2015, 1-50.

16 DTE Energy. Smart Currents Dynamic Peak Pricing Pilot - Final Evaluation Report. August 2014, 1-121.

17 Blumsack AS, Beraldi A, Mountain G, et al. Load Impact Analysis of Green Mountain Power Critical Peak Events, 2012 and 2013. March 2015, 1-62.

18 Lakeland Electric. Lakeland Electric Consumer Behavior Study Final Evaluation Report. April 2015, 1-52.

19 GDS Associates. Marblehead Municipal Light Department Energysense CPP Pilot Final Evaluation Report. June 2013, 1-47.

20 Energy Center of Wisconsin. Minnesota Power's Advanced Metering Infrastructure Project AMI Behavioural Research Pilot Interim Results from a Consumer Enhanced Feedback Pilot. Prepared by Energy Center of Wisconsin for Minnesota Power. March 2014, 1-51.

21 NV Energy. Nevada Dynamic Pricing Trial Final Report: An evaluation of NV Energy's Choose When You Use Program. October 2015, 1-113.

22 Potter J, George S, Jiminez L. SmartPricing Options Final Evaluation: The Final report on pilot design, implementation and evaluation of the Sacaramento Municipal Utility District's Consumer Behavior Study. September 2014, 1-192.

23 Bleything S, Blumsack S, Hines P, Morris L. Vermont Electric Cooperative Consumer Behavior Study Year 2 Final Report - the Effect of Variable Peak Pricing on Electricity Demand. June 2015, 1-55.

24 Bourne T, Watson M. Sunshine Tariff - Customer Recruitment Learning Report. Western Power Distribution Innovation. 2016. https://www.westernpowerinnovation.co.uk/ Document-library/2017/Sunshine-Tariff/Final-Sunshine-Tariff-Customer-Recruitment.aspx.

25 Star A, Isaacson M, Haeg D, Kotewa L, CNTenergy. The Dynamic Pricing Mousetrap: Why Isn't the World Beating Down Our Door? ACEEE Summer Study on Energy Efficiency in Buildings, 2010: 257-268.

26 Verhagen E, Ketter W, Rook L, van Dalen J. The impact of framing on consumer selection of energy tariffs. In: 2012 International Conference on Smart Grid Technology, Economics and Policies (SG-TEP). IEEE; 2012:1-5.

27 K. Stenner, E. Frederiks, E. V Hobman, and S. Meikle, “Australian Consumers' Likely Response to Cost- Reflective Electricity Pricing,” 2015.

\section{References}

[1] Carreiro AM, Jorge HM, Antunes CH. Energy management systems aggregators: a literature survey [no. January]. Renew Sustain Energy Rev 2017;73:1160-72.

[2] US Department of Energy, Final Report on Customer Acceptance, Retention and Response to Time-Based Rates from the Consumer Behavior Studies,; 2016.

[3] Newsham GR, Bowker BG. The effect of utility time-varying pricing and load control strategies on residential summer peak electricity use: a review. Energy Policy . 2010;38(7):3289-96.

[4] Faruqui A, Sergici S. Arcturus: international evidence on dynamic pricing. Electr J 2013;26(7):55-65.

[5] Frontier Economics and Sustainability First, Demand Side Response in the domestic sector- a literature review of major trials, London; 2012.

[6] Consumer Focus, From devotees to the disengaged, 2012 pp. 1-19.

[7] Crossley D. Task 15 - Case Study - TEMPO Electricity Tariff - France. 2009 pp. 1-2.

[8] Johnson E, Goldstein D. Do defaults save lives? Science 2003;302(5649):1338-9.

[9] Pichert D, Katsikopoulos KV. Green defaults: information presentation and pro-environmental behaviour. J Environ Psychol . 2008;28(1):63-73.

[10] Thaler R, Sunstein C. Nudge: improving decisions about health, wealth and happiness. Yale University Press; 2008.

[11] Carroll GD, Choi JJ, Laibson D, Madrian BC, Metrick A. Optimal defaults and active decisions. Q J Econ . 2009;124(4):1639-74.

[12] Grant MJ, Booth A. A typology of reviews: an analysis of 14 review types and associated methodologies. Health Inform Libr J 2009;26(2):91-108.

[13] Liberati A, Altman DG, Tetzlaff J, Mulrow C, Gøtzsche PC, Ioannidis JPA, Clarke M, Devereaux PJ, Kleijnen J, Moher D. The PRISMA statement for reporting systematic reviews and meta-analyses of studies that evaluate healthcare interventions: explanation and elaboration. BMJ 2009;339:b2700.

[14] Fell MJ, Nicolson M, Huebner G, Shipworth D. Is it time? Consumers and time of use electricity tariffs: Trialling the effect of tariff design and marketing on consumer demand for demand-side response tariffs, London; 2015.

[15] Fell MJ. Taking charge: perceived control and acceptability of domestic demandside response. UCL (University College London); 2016.

[16] Hobman EV, Frederiks ER, Stenner K, Meikle S. Uptake and usage of cost-reflective electricity pricing: insights from psychology and behavioural economics. Renew Sustain Energy Rev 2016;57:455-67.

[17] Stenner K, Frederiks E, Hobman EV, Meikle S. Australian Consumers' Likely Response to Cost- Reflective Electricity Pricing,; 2015.

[18] Dütschke E, Paetz A-G. Dynamic electricity pricing-which programs do consumers prefer? Energy Policy . 2013;59:226-34.

[19] Kontapantelis E, Reeves D. metaan - random-effects meta-analysis - Stata Journal.pdf. Stata J 2010;10(3):395-407.

[20] Kelley GA, Kelley KS. Statistical models for meta-analysis: a brief tutorial. World J Methodol 2012;2(4):27-32.

[21] Schwartz D, Bruine de Bruin W, Fischhoff B, Lave L. Advertising energy saving programs: the potential environmental cost of emphasizing monetary savings. J Exp Psychol Appl 2015;21(2):158-66.

[22] Verhagen E, Ketter W, Rook L, van Dalen J. The impact of framing on consumer selection of energy tariffs, In: Proceedings of international conference on smart grid technology, economics and policies (SG-TEP); 2012, p. 1-5.

[23] Buryk S, Mead D, Mourato S, Torriti J. Investigating preferences for dynamic electricity tariffs: the effect of environmental and system benefit disclosure. Energy Policy 2015;80:190-5.

[24] Hartway R, Price S, Woo CK. Smart meter, customer choice and profitable time-ofuse rate option. Energy 1999;24(10):895-903.

[25] Lutzenhiser S, Peters J, Moezzi M, Woods J. Beyond the Price Effect in Time-of-Use Programs : Results from a Municipal Utility Pilot , 2007-2008, International Energy Programme Evaluation Conference 2009, 2010, p. 2007-2008.

[26] Lakeland Electric, Lakeland Electric Consumer Behavior Study Final Evaluation Report,; 2015.

[27] Charles River Associates, Primer on Demand-Side Management with an Emphasis 
on Price-Responsive Programs - Report prepared for The World Bank, Oakland, California; 2005.

[28] BEIS, Smart Energy Research - BEIS Consumer Panel,; 2016.

[29] Nicolson M, Huebner G, Shipworth D. Are consumers willing to switch to time of use electricity tariffs? The importance of loss-aversion and electric vehicle owner ship. Energy Res Soc Sci 2017;23:82-96.

[30] Stenner K. Understanding likely customer response to future electricity tariff designs: Insights from Behavioural Economics,; 2015.

[31] Sheeran P. Intention - behavior relations: a conceptual and empirical review [no. February]. Eur Rev Soc Psychol 2002;3283:1-30.

[32] Whitehead JC, Blomquist GC. The use of contingent valuation in benefit-cost analysis. In: Alberini A, Kahn JR, editors. Handbook on Contingent Valuation. Cheltenham, UK: Edward Elgar; 2006.

[33] Sheppard BH, Hartwick J, Warshaw PR. The theory of reasoned action: a metaanalysis of past research with recommendations for modifications and future research. J Consum Res 1988;15(3):325-43.

[34] Morwitz VG, Steckel JH, Gupta A. When do purchase intentions predict sales? Int J Forecast . 2007;23(3):347-64.

[35] Defeuilley C. Retail competition in electricity markets. Energy Policy . 2009;37(2):377-86.

[36] Fell MJ, Shipworth D, Huebner GM, Elwell CA. Public acceptability of domestic demand-side response in Great Britain: the role of automation and direct load control. Energy Res Soc Sci 2015;9:72-84.

[37] Potter J, George S, Jiminez L. SmartPricing Options Final Evaluation: The Final report on pilot design, implementation and evaluation of the Sacaramento Municipal Utility District's Consumer Behavior Study,; 2014.

[38] Phillips R, Owen G, Ward J. Project Lessons Learned from Trial Recruitment: Customer - Led Network Revolution Trials,; 2013.

[39] Bourne T, Watson M. Sunshine tariff - customer recruitment learning report,; 2016.

[40] Carmichael R, Schofield J, Woolf M, Bilton M, Ozaki R, Strbac G. Residential consumer attitudes to time-varying pricing Report 2 for the Low Carbon London LCNF project, 2014 pp. 1-93.

[41] Nicolson M, Huebner GM, Shipworth D, Elam S. Tailored emails prompt electric vehicle owners to engage with tariff switching information [no. May]. Nat Energy 2017;2:1-6.
[42] Whitaker G, Wardle R, Barteczko-Hibbert C, Matthews P, Bulkeley H, Powells G. CLNR Insight Report: Domestic Time of Use Tariff: A comparison of the time of use tariff trial to the baseline domestic profiles,; 2013.

[43] BEIS, Smart meter roll-out cost benefit analysis - Part I,; 2016.

[44] Ofgem, The Innovation Link Open Letter,; 2017.

[45] Commission for Energy Regulation, CER National Smart Metering Programme Managing the Transition to Time-of-Use Tariffs, Dublin; 2015.

[46] Harper H. Applying Behavioural Insights to Organ Donation: preliminary results from a randomised controlled trial,; 2012.

[47] Social and Behavioral Sciences Team, Social and Behavioral Sciences Team Annual Report Executive Office of the President National Science and Technology Council,; 2015.

[48] Hallsworth M, List JA, Metcalfe RD, Vlaev I, The Behavioralist As Tax Collector: Using Natural Field Experiments to Enhance Tax Compliance, Cambridge, MA, 20007, Mar. 2014.

[49] Haynes LC, Green DP, Gallagher R, John P, Torgerson DJ. Collection of delinquent ines: an adaptive randomized trial to assess the effectiveness of alternative text messages. J Policy Anal Manag . 2013;32(4):718-30.

[50] Schultz PW, Nolan JM, Cialdini RB, Goldstein NJ, Griskevicius V. The constructive, destructive, and reconstructive power of social norms: research article. Psychol Sci 2007; 18(5):429-34.

[51] Cialdini RB, Martin SJ, Goldstein NJ. Small behavioral science-informed changes can produce large policy-relevant effects. Behav Sci Policy 2015;1(1)

[52] Andreoni J. Cooperation in public-goods experients: kindness or confusion? Am Econ Rev 1995;85(4):891-904.

[53] Wilson C, Dowlatabadi H. Models of decision making and residential energy use. Annu Rev Environ Resour . 2007;32(1):169-203.

[54] Frederiks ER, Stenner K, Hobman EV. Household energy use: applying behavioural economics to understand consumer decision-making and behaviour. Renew Sustain Energy Rev 2015;41:1385-94.

[55] Train KE, McFadden DL, Goett AA. Consumer \{Attitudes\} and \{Voluntary\} \{Rate \{Schedules\} for \{Public\} \{Utilities\}. Rev Econ Stat 1987;69(3):383-91.

[56] Raw G, Ross D, Energy Demand Research Project: Final Analysis, Jun. 2011.

[57] Neenan B, Patton M. First Energy's Smart Grid Investment Grant Consumer Behavior Study Final Evaluation; 2015. 